\title{
Avaliação do cloreto de benzalcônio no controle da ferrugem (Hemileia vastatrix Berk. \& Br.) do cafeeiro (Coffea arabica L.)
}

\author{
Adriana Novais Martins ${ }^{1}$, Arlindo Pinheiro da Silveira ${ }^{2}$, Edson Luiz Furtado ${ }^{3}$
}

\begin{abstract}
1APTA Regional do Médio Paranapanema, Rod. SP 333 (Assis - Marília) km397, CEP 19800-000, Assis, SP, e.mail: adrianamartins@apta.sp.gov.br; ${ }^{2}$ SM Consultoria e Experimentação Agríc ola Ltda., Av. Cristo Rei, 52, Marília, SP. e.mail: arlindo.pinheiro@flash.tv.br; ${ }^{3}$ UNESP, Faculdade de Ciências Agronômicas, Departamento de Produção Vegetal, CP 237, CEP 18.603-970, Botucatu, SP. e.mail: elfurtado@fca.unesp.br Autor para correspondência: Adriana Novais Martins Data de chegada: 28/11/2005. Aceito para publicação em: 27/03/2007
\end{abstract}

\section{RESUMO}

Martins, A.N.; Silveira, A. P. da; Furtado, E.L. Avaliação do Cloreto de Benzalcônio no controle da ferrugem (Hemileia vastatrix Berk. \& Br.) do cafeeiro (Coffea arabica L.) Summa Phytopathologica, v.35, n.2, p. 143-145, 2009

Com o objetivo de avaliar o desempenho do clore to de benzalcônio em programas de tratamento com fungicida cúprico e epoxiconazole, no controle da ferrugem (Hemileia vastatrix Berk. \& Br.) do cafeeiro (Coffea arabica L.), foi conduzido um experimento no município de Garça, SP. Foram utilizados cafeeiros do cultivar Mundo Novo 388-6-20, plantados no espaçamento de 4,0 x 1,5 m (1.666 covas/ha). Os resultados obtidos mostraram que aplicação de um fungicida triazol foi fundamental para o controle da ferrugem, considerada a principal doença da cultura. Programa de tratamento com cloreto de benzalcônio aplicado entre dezembro e fevereiro, em intervalos de trinta dias associado ao epoxiconazole aplicado em março, apresentou bom resultado no controle da doença. A utilização de oxicloreto de cobre associado ao epoxiconazole proporcionou maior retenção foliar. Estudos complementares devem ser realizados em outras localidades e lavouras com diferentes cargas pendentes, para que o cloreto de benzalcônio possa ser incluído como componente no manejo da ferrugem do cafeeiro.

Palavras-chave adicionais: manejo fitossanitário; efeito tônico; fungicidas triazóis; fitotoxidez

\section{ABSTRACT}

Martins, A.N.; Silveira, A. P. da; Furtado, E.L. Evaluation of Benzalkonium Chloride on the control of coffee leaf rust. Sum ma Phytopathologica, v.35, n.2, p.145-145, 2009

With the objective to evaluate the benzalkonium chloride performance in programs of treatment with copper fungicide and epoxiconazol to control coffee leaf rust (Hemileia vastatrix Berk. \& Br.), an experiment was lead in the Garça city, SP. They had been evaluated coffee of cultivar 'Mundo Novo 388-6-20' spaced at 4,0 x $1,5 \mathrm{~m}$. The results obtained showed that the application of triazol fungicide was important to control coffee leaf rust, considered the main disease of the culture. Programs of control with benzalkonium chloride applied between December and February, in intervals of thirty days associate to the epoxiconazol applied in March, presented good result in the control of the disease. The use of copper fungicide associated to the epoxiconazol provided great foliar retention. Complemental studies should be accomplished in other places and crops with differents productions, so that the benzalkonium chloride can be included as component in the control of the coffee leaf rust.

Keywords: tonic effect; fungicides; wield programs

A ferrugem (Hemileia vastatrix Berk. \& Br.) é considerada a doença mais importante na cultura do cafeeiro. Por ser um processo dinâmico e dependente de vários fatores, principalmente àqueles relacionados às condições climáticas, fisiológicas da planta e carga pendente, a infecção ocorre de maneira não padronizada, fazendo com que a curva de progresso da doença varie de ano para ano em intensidade e agressividade (6).

O Cloreto de Benzalcônio é uma amônia quaternária que atua internamente na planta através da indução de mecanismos de defesa como o aumento do teor de compostos fenólicos, além das síntese de â-1,3-glucanase e quitinases, no tecido vegetal, conferindo uma proteção local, não evidenciando a proteção sistêmica. Há também uma ação direta sobre o patógeno Hemileia vastatrix pela inibição da germinação dos es poros do fungo e da formação de apressórios $(8,7)$.
Este produto pode apresentar um efeito fungistático, suprimindo a esporulação das pústulas de ferrugem, na maioria dos casos, de forma temporária (1).

Com o objetivo de avaliar o desempenho do Cloreto de Benzalconnio associado a programas de controle fitossanitário das doenças foliares do cafeeiro, foi instalado um ensaio no Campo Experimental "Dr. Alcides Carvalho", na Cooperativa dos Cafeicultores de Garça GARCAFÉ, no Município de Garça, Estado de São Paulo, em um lote do cultivar Mundo Novo 388-6-20, com 8 anos de idade, plantado no espaçamento de 4,0 x 1,5 m, 2 plantas por cova, totalizando 1666 covas / ha, em ano de safra pendente elevada.

O delineamento experimental adotado foi emblocos ao acaso, com 8 tratamentos e 4 repetições. Cada parcela foi formada por 11 covas, sendo avaliadas as 7 centrais. Os tratamentos foram: A - 
Tabela 1. Efeito dos programas de tratamento sobre a porcentagem de infecção da ferrugem, número de folhas remanescentes (NFR) por ramo em avaliação pré-colheita e área abaixo da curva de progresso da doença (AACPD). Garça, SP.

\begin{tabular}{|c|c|c|c|c|c|c|c|}
\hline \multirow{2}{*}{ Tratamentos $^{1}$} & \multicolumn{5}{|c|}{ Infecção (\%) } & \multirow{2}{*}{$\frac{\text { NFR }}{\text { junho }}$} & \multirow{2}{*}{ AACPD } \\
\hline & fevereiro & março & abril & maio & junho & & \\
\hline A - Testemunha & $19,5 \mathrm{a}^{*}$ & 37,0 a & 57,5 a & $65,0 \mathrm{a}$ & 45,0 a & $6,5 \mathrm{a}$ & 5752,5 a \\
\hline B - EP (dez $75 \mathrm{~g}$ i.a./ha + mar 50g i.a./ha) + OC (jan/fev) & $1,0 \quad \mathrm{c}$ & $3,5 \mathrm{~d}$ & 2,5 & $0,0 \quad \mathrm{c}$ & $0,0 \quad \mathrm{~d}$ & $14,8 \quad \mathrm{c}$ & 195,0 \\
\hline $\mathrm{C}-\mathrm{OC}$ (dez a abr, interv. 30 dias) & $5,0 \mathrm{~b}$ & 9,5 cde & 8,5 cde & $16,5 \mathrm{~b}$ & $3,5 \mathrm{c}$ & $12,7 \mathrm{bc}$ & $1162,5 \mathrm{bcd}$ \\
\hline $\mathrm{D}-\mathrm{CB}$ (dez a fev, interv. 30 dias $)+\mathrm{EP}$ (mar 75g i.a./ha) & $3,0 \mathrm{bc}$ & 8,0 cde & 4,5 de & $0,0 \mathrm{c}$ & $0,0 \quad \mathrm{~d}$ & $12,0 \mathrm{bc}$ & 420,0 \\
\hline $\mathrm{E}-\mathrm{CB}$ (dez a fev, intervalos 30 dias) $+\mathrm{OC}$ (mar/abr) & $2,5 \mathrm{bc}$ & 8,5 cde & $17,0 \mathrm{bc}$ & $16,0 \mathrm{~b}$ & $10,5 \mathrm{~b}$ & $10,4 \quad b$ & 1440,0 bc \\
\hline $\mathrm{F}-\mathrm{CB}(\mathrm{fev})+\mathrm{EP}(\operatorname{mar} 75 \mathrm{~g}$ i.a. $/ \mathrm{ha})$ & $3,5 \mathrm{bc}$ & $14,5 \mathrm{bc}$ & $11,5 \mathrm{bcd}$ & $4,0 \quad \mathrm{c}$ & $0,0 \quad \mathrm{~d}$ & $10,9 \mathrm{bc}$ & 922,5 \\
\hline $\mathrm{G}-\mathrm{CB}(\mathrm{dez} / \mathrm{fev})+\mathrm{OC}(\mathrm{mar} / \mathrm{abr})$ & $4,5 \mathrm{bc}$ & $13,0 \mathrm{bcd}$ & $25,5 \mathrm{~b}$ & $20,0 \mathrm{~b}$ & $5,0 \quad \mathrm{c}$ & $9,5 \mathrm{ab}$ & $1897,5 \quad b$ \\
\hline $\mathrm{H}$ - EP (mar $75 \mathrm{~g}$ i.a./ha) + OC (abr) & $15,7 \mathrm{a}$ & $28,0 \mathrm{ab}$ & $16,5 \mathrm{bc}$ & $0,0 \quad \mathrm{c}$ & $3,5 \quad \mathrm{c}$ & $11,2 \mathrm{bc}$ & $1638,5 \mathrm{bc}$ \\
\hline C. V. $(\%)$ & 20,35 & 22,88 & 17,76 & 19,74 & 16,42 & 7,11 & 19,97 \\
\hline
\end{tabular}

* médias seguidas por letras distintas diferem entre si ao nível de $5 \%$ de probabilidade pelo teste de Tukey

${ }^{1} \mathrm{EP}$ - epoxiconazole; $\mathrm{OC}$ - oxicloreto de cobre; $\mathrm{CB}$ - cloreto de benzalcônio. Todas as aplicações da oxicloreto de cobre foram feitas utilizando-se $1500 \mathrm{~g}$ i.a./ha e as de cloreto de benzalcônio, utilizando-se $1200 \mathrm{~g}$ i.a./ha.

Testemunha; B - epoxiconazole (dezembro a $75 \mathrm{~g}$ i.a./ha e março a 50 g i.a/ha) + oxicloreto de cobre (janeiro e fevereiro); $\mathbf{C}$ - oxicloreto de cobre (dezembro a abril, em intervalos de 30 dias); D - cloreto de benzalcônio (dezembro a fevereiro, em intervalos de 30 dias) + epoxic onazole (março a $75 \mathrm{~g}$ i.a./ha); $\mathbf{E}$ - cloreto de benzalcônio (dezembro a fevereiro, em intervalos de 30 dias) + oxicloreto de cobre (março e abril); F -- cloreto de benzalcônio (fevereiro) + epoxiconazole (março a $75 \mathrm{~g}$ i.a./ha); $\mathbf{G}$ - cloreto de benzalcônio (dezembro e fevereiro) + oxicloreto de cobre (março e abril); $\mathbf{H}$ - epoxiconazole (março a 75 g i.a./ha) + oxicloreto de cobre (abril). As doses de oxicloreto de cobre foram de $1500 \mathrm{~g}$ i.a./ha e as de cloreto de benzalcônio, $1200 \mathrm{~g}$ i.a./ha.

Foram estimados a incidência da doença pelaporcentagem de folhas infectadas com ferrugem, no período de fevereiro a junho, e o número de folhas remanescentes por ramo em pré-colheita, no mês de junho, sendo que a colheita foi realizada no mês seguinte (julho). A incidência da doença também foi avaliada em termos de Área Abaixo da Curva de Progresso da Doença (AACPD). As avaliações referentes à porc entagem de folhas infectadas com ferrugem foram realizadas em amostras de 50 folhas por parcela, coletadas em intervalos de 30 dias, no terço médio das plantas. Para efeito de avaliação, os dados foram submetidos à análise de variância utilizando-se o software estatístico SAS, após serem transformados em $\sqrt{x+0.5}$, e para a comparação entre médias foi utilizado o teste de Tukey a $5 \%$ de significância. Todas as pulverizações foram realizadas com pulverizador costal motorizado, com bico tipo cone, com um consumo de calda 400 litros/ ha.

De acordo com os resultados obtidos, observou-se que a doença apresentou evolução até o mês de maio, quando atingiu o máximo de folhas infectadas (Tabela 1).

Os tratamentos que receberam aplicação de epoxiconazole em março (Tratamentos B, D, F e $\mathbf{H}$ ) apresentaram porcentagens de infecção próximas ou iguais a zero no mês de maio. Em junho a porcentagem de folhas infectadas apresentou uma diminuição generalizada provavelmente devido à queda das folhas doentes.

O tratamento B [epoxiconazole (dezembro a $75 \mathrm{~g}$ i.a./ha e março a $50 \mathrm{~g}$ i.a/ha) + oxicloreto de cobre (janeiro e fevereiro)] apresentou porc entagens de infecção muito baixas durante todo o experimento, apresentando o menor valor de AACPD, e maior enfolhamento, provavelmente porque recebeu uma primeira aplicação de epoxiconazole em dezembro, que pode ter mantido o potencial de inóculo inicial de $H$. vastatrix baixo, favorecendo o desenvolvimento vegetativo das plantas. Observando-se o tratamento $\mathbf{D}$ [cloreto de benzalcônio (dezembro a fevereiro, em intervalos de 30 dias) + epoxiconazole (março a $75 \mathrm{~g}$ i.a./ha)] verifica-se que as ap licações de cloreto de benzalcônio auxiliaram na redução da incidência da doença, provavelmente por causa da ação de indução local sobre a germinação dos uredósporos (7), concordando com resultados obtidos por Almeida et al. (5) e Silva et al. (9).

Todos os tratamentos que não receberam o epoxiconazole (Tratamentos $\mathbf{C}, \mathbf{E}$ e $\mathbf{G}$ ) resultaram em porcentagens mais elevadas de incidência da doença, quando comparados aos que receberam aplicações do produto. Este comportamento é melhor visualizado através dos resultados de AACPD (Tabela 1), onde observa-se o melhor desempenho dos tratamentos com aplicação do fungicida triazol.

Entre todos os tratamentos em que não foi realizada a aplicação de epoxiconazole, o G [cloreto de benzalcônio (dezembro e fevereiro) + oxicloreto de cobre (março e abril)] foi o que apresentou o pior desempenho no decorrer das avaliações, alcançando índice de infecção de $25,5 \%$ no mês de abril. Este índice refletiu no baixo número de folhas remanescentes por ramo em pré-colheita, no mês de junho.

Em todos os tratamentos em que foi aplicado o cloreto de benzalcônio nos meses de dezembro a fevereiro, com intervalos de 30 dias (D e E), verificou-se que o produto auxiliou no controle da doença, concordando com os resultados obtidos por Almeida et al. (2). A incidência da doença foi mantida emíndices relativamente baixos, equiparados ao apresentado pelo tratamento onde se aplic ou somente o fungicida cúprico, caindo este desempenho quando o produto foi aplicado a intervalos de 45 dias. Este fato evidencia o potencial do produto para ser utilizado em programas de manejo da ferrugem do cafeeiro, desde que haja a inclusão de um fungicida triazol, como por exemplo, o epoxic onazole, conforme resultados obtidos por Silva et al. (9), Almeida et al. (3) e Almeida et al. (4).

Os resultados demonstraram que os programas de tratamentos que não receberam aplicações do fungicida triazol mostraram resultados inferiores aos demais tratamentos, diferindo, entretanto, da Testemunha. Este fato evidenciou uma tendência de ação diferencial do cloreto de benzalcônio em programas de controle da ferrugem do cafeeiro. Entretanto, apesar das evidências iniciais do potencial do produto para o controle da doença, esteainda deve ser avaliado em experimentos realizados em outras localidades e em lavouras com diferentes cargas pendentes, para que possa ser incluído como um componente eficaz no manejo da ferrugem do cafeeiro. 


\section{REFERÊNCIAS BIBLIOGRÁFICAS}

1. Almeida, S.R.; Matiello, J.B.; Ferreira, R.A. Estudo preliminar sobre o efeito do Fegatex (amônia quaternária) sobre a ferrugem do cafeeiro. In: Congresso Brasileiro de Pesquisas Cafeeiras, 23., 1997. Manhuaçu. Anais. Rio de Janeiro: MAPA/PROCAFÉ, 1997. p. $71-72$.

2. Almeida, S.R.; Matiello, J.B.; Ferreira, R.A. Efeito do Fegatex (amônia quaternária) em diferentes concentrações e associação com adjuvante no controle da ferrugem do cafeeiro. In: Congres so Brasileiro de Pesquisas Cafeeiras, 24., 1998, Poços de Caldas. Anais. Rio de Janeiro: MAPA/PROCAFÉ, 1998. p. 51-52.

3. Almeida, S. R.; Matiello, J.B.; Ferreira, R.A. Efeito da associação de fungicidas sistêmicos e cúpricos com o produto Fegatex no controle da ferrugem do cafeeiro. In: Congresso Brasileiro de Pesquisas Cafeeiras, 25., 1999, Franca. Anais. Rio de Janeiro: MAPA/PROCAFE, 1999.p. 197-198.

4. Almeida, S.R.; Matiello, J.B.; Ferreira, R.A. Combinação de Fegatex (Amônia quaternária, Cloreto de Benzalcônio) com vários fungicidas no controle da ferrugem do cafeeiro. In: Congresso Brasileiro de Pesquisas Cafeeiras, 26., 2000, Marília. Anais. Rio de Janeiro: MAPA/PROCAFÉ, 2000. p. 30-31.

5. Almeida, S. R.; Matiello, J. B.; Ferreira, R. A.; Perez, Rodrigo M.
Controle da ferrugem do cafeeiro com o fungicida Flamenco Fluquinconazole e combinações de cobre e triazóis com Fegatex. In: Congresso Brasileiro de Pesquisas Cafeeiras, 28., 2002, Caxambu. Anais. Rio de Janeiro : MAPA/PROCAFÉ, 2002., p. $133-134$.

6. Godoy, C.V.; Bergamin Filho, A.; Salgado, C.L. Doenças do cafeeiro. In: Kimati, H. et al. (Ed.) Manual de Fitopatologia, 3. ed. São Paulo, Ceres. 1997. p.184-200. vol. 2: Doenças das Plantas Cultivadas.

7. Guzzo, S.D.; Harakava, R.; Kida, K.; Martins, E.M.F.; Roveratti, D.S. Proteção de cafeeiros contra Hemileia vastatrix por Cloreto de Banzalcônio (composto de amônio quaternário). Summa Phytopathologica, Jaboticabal, v. 25, n. 4, p. 339-345, 1999.

8. Harakava, R.; Guzzo, D.; Roveratti, D.S. Efeito de um composto de amônio quaternário na proteção de plantas de café à ferrugem. Summa Phytopathologica, Jaguariúna, v. 20, n. 1, p. 53, 1994. (resumo 104).

9. Silva, A.C.; Salgado, L.O.; Gitirana Neto, J.; Santos E.E.V.; Dias, W.H. Estudo da associação do produto Biopirol e Fegatex com o triazol Horizon visando controle da doença Hemileia vastatrix (ferrugem), na cultura do cafeeiro (Coffea arabica L.). In: Congresso Brasileiro de Pesquisas Cafeeiras, 28., 2002, Caxambu. Anais. Rio de Janeiro: MAPA/PROCAFÉ, 2002. p. 287-289. 